\title{
A Resilient and Scalable novel Protocol for Wireless Sensor Networks -Maximum Energy Clustering (MEC)
}

\author{
Abhinav Garg \\ Associate Professor, National Institute of Fashion Technology, Hyderabad India \\ abhinavarungarg1@gmail.com \\ Dr. Manisha Jailia \\ Associate Professor, Banasthali Vidyapith, Rajasthan India \\ manishajailia@yahoo.co.in
}

\begin{abstract}
Wireless Sensor Networks (WSNs) refer to a collection of innumerous sensing de-vices and actuators that form a network to sense the environment or physical conditions and relay the information back to the base station. When the network size grows, clustering becomes intrinsic for the network and many clustering protocols have been studied so far but it is observed that they follow a probabilistic CH election process that gives rise to uncertainties in the algorithm like variable cluster size, uneven node degree of the CHs etc. Here a resilient and scalable novel protocol Maximum Energy Clustering Protocol (MEC) is proposed for the WSN which takes a deterministic route to find $\mathrm{CH}$ nodes and optimal number of CHs in a round, rather than probabilistic approach followed by its counterparts. Results are compared to a well-known protocol DEEC (Distributed energy efficient clustering) and it is examined that Maximum Energy Clustering Protocol (MEC) outperforms DEEC as it follows a fair CH election process. MEC is able to attain a significant reduction in network traffic besides utilizing the network to its fullest capacity. Results show that Maximum Energy Clustering Protocol (MEC) outperforms its predecessor DEEC in terms of energy efficiency, life span of network and controlling intra cluster traffic in the network.
\end{abstract}

Keywords: IoT (Internet of Things), Redundancy, Wireless Sensor Networks (WSN's), Energy Efficiency, Clustering, Heterogeneous networks, Network Lifetime.

\section{Introduction}

In the recent times, the smart city concept has created a buzz across the continents. ICT, networking, IoT and Cloud computing are the pillars of a smart city[2]. With the explosion of

Smart Cities, there will be a growing need to have efficient protocols to manage these dense underlying networks which keeps the whole system at place. Smarter Data Science and technologies are required for handling innumerous data produced by Wireless Sensor networks and ICT devices. [2].

Wireless Sensor Networks (WSNs) stands for the collection of innumerous sensing devices and actuators that form a network to sense the environment or physical conditions and relay the information back to the aggregators(BS) or cloud servers. They may also be referred to as IOT networks. When the network size grows, clustering becomes intrinsic for the governance of the network. Large networks are generally divided into clusters where every cluster elects a Cluster $\operatorname{Head}(\mathrm{CH})$. CHs usually have greater data processing abilities than the other normal nodes. The normal sensor nodes constantly monitor the physical conditions and relay data to their respective $\mathrm{CH}$. Sometimes nodes in a network are very closely placed and all of them sense the same data and send it to $\mathrm{CH}$. It might also happen when the nodes are mobile. This repetition of data results in a lot of energy and bandwidth going waste.

Many conventional clustering protocols have been studied in the histories which have contributed to the growth and expansion of WSNs. In dense sensor networks, the motes are closely packed to each other. The point where sensing zones of different sensors overlap, it is observed that there are high similarities in the readings produced by enormous time stamped data generated by these networks. [1].

One such fundamental algorithm is self-organizing LEACH [3] which henceforth laid the foundation of many WSN protocols. LEACH gave the idea of dividing the entire cycle of network formation and data transmission in two phases:- Setup phase and steady phase. Set up phase is responsible for handshake i.e. cluster formation whereas actual data transmission takes place in steady phase. Sensor nodes elect themselves as $\mathrm{CH}$ on the basis of a probability. Though a randomized rotation of $\mathrm{CH}$ responsibility takes place to assure the participation of all the nodes and distribution of the energy consumption amongst nodes still there are high chances of the same node becoming $\mathrm{CH}$ again, resulting in energy drain of few nodes. $\mathrm{CH}$ election process in LEACH is highly randomized, so total number of $\mathrm{CHs}$ in a round varies arbitrarily leading to uneven load distribution on the $\mathrm{CH}$ [27]. 
Many authors suggested improvements over LEACH which is discussed in the next section of this paper. It has been observed that these protocols have some issues like instability of network, irregular cluster head $(\mathrm{CH})$ count, highly fluctuating node degrees of $\mathrm{CH}$, redundancy of data and packet loss. WSN protocols studied so far are highly probabilistic. As proposed in LEACH [3], DEEC [17], TEEN [23], HEED [16], a typical cycle in these protocols start with nodes electing themselves as Cluster Head $(\mathrm{CH})$ depending on probability and random selected variables. Some of the drawbacks observed in these techniques are:

- CHs are randomly and unevenly distributed. [28] CH election is totally based on a stochastic parameter which is compared with a randomly generated number. [29]

- During $\mathrm{CH}$ selection, parameters like Residual energy of the nodes, number of alive nodes in the network, distances between the nodes in the network are not considered.

- Sizes of the clusters vary abruptly in every round as it is decided at random. Variation in number of CHs per round gives rise to variation in the node degree (No. of non- $\mathrm{CH}$ nodes per cluster) of each $\mathrm{CH}$.

- At the time, when the no. of CHs are low, Single hop communication or direct contact with Base Station (BS) increases, giving rise to more energy consumption and congestion of the network.

This approach, MEC (Maximum Energy Clustering) protocol for WSNs commits to address and resolve the above stated shortcomings in the protocols mentioned in the literature. This experiment aims to resolve these issues and design an Energy Aware Resilient and Scalable protocol for the emergent WSN's. The central idea of proposed protocol MEC(Maximum energy Clustering) is to control number of $\mathrm{CHs}$ which should be directly dependent on the No. of alive nodes in the network. As discussed in the results section, MEC protocol accomplishes to aggrandize the network utilization while reducing the redundancy at same time using event driven programming.

\section{Literature Review}

Numerous clustering and data aggregation algorithms for Wireless sensor networks (WSNs) have been proposed in the history. The focal point of these algorithms has been to make them energy efficient and improve overall network efficiency. In this paper we will focus on clustering protocols as the proposed work is centered on the same.

W. Heinzelman et. al. [3], presented a protocol, low-energy adaptive clustering hierarchy (LEACH) which was based on a distributed, self-organizing cluster formation technique. New cluster heads are nominated in every round to evenly distribute the network load. It ensures that each node gets a fair chance to become a cluster head depending on a probability (p). A node will be selected as a $\mathrm{CH}$ if it reaches the threshold which is calculated as

$$
T(n)= \begin{cases}\frac{p}{1-p \bmod \left(r, \frac{1}{p}\right)} & \text { if } n \in G \\ 0 & \text { otherwise }\end{cases}
$$

Where $\mathrm{p}$ is Optimum Election Probability that a node can turn out to be cluster head, $r$ is the index of current round, $\mathrm{N}$ is the number of nodes, $\mathrm{G}$ is the group of nodes that are eligible to serve as cluster head in that round. CHs take data from all the normal nodes, aggregate and forward it to the base station (BS). However as mentioned, LEACH [3] becomes unstable with the increase in heterogeneity.

A lot of protocols have been proposed on the lines of LEACH like A-LEACH [4] , LEACH-B [5], S-LEACH [6] and M-LEACH [7], Multi-level Hierarchal LEACH [8], MR-LEACH [9], Q-LEACH [10].

D. Mahmood et al proposed a variant of LEACH named as MOD-LEACH [11], with two transmission levels and different amplification energies for inter cluster, intra cluster communication. They further enhanced and compared the scheme with threshold imperative version of MOD-LEACH i.e. MODLEACH with hard threshold (MODLEACHHT) and MODLEACH with soft threshold (MODLEACHST). The technique achieves longer network lifetime because of control on the transmissions.

The $\mathrm{CH}$ election process is still same as in LEACH [3] and its variants. CH election is totally dependent on probability and a randomly generated number that leads rise to uneven node degree of the $\mathrm{CH}$.

G. Smaragdakis et. al. Proposed a protocol SEP [12]. It is a heterogeneous network protocol where nodes have dissimilar energy levels. CHs are elected depending on the weight of the probability of election and the residual energy of each node. The network supports two level heterogeneity where some nodes called superior nodes have slightly extra energy in comparison to Normal nodes.

$$
\begin{aligned}
& \text { No. of advanced nodes }=m^{*} n \\
& \quad \text { No. of normal nodes }=(1-m) * n
\end{aligned}
$$

Where $\mathrm{n}$ denotes the total number of nodes and $\mathrm{m}$ represents the percentage of nodes which possess $\alpha$ times extra energy than the others. 


$$
T_{\text {total }}=n \cdot E_{o}(1+\alpha \cdot m)
$$

Though there is an increase in the overall energy of the network by $1+\alpha \cdot \mathrm{m}$ times, the $\mathrm{CH}$ election process still remains the same with uneven cluster size.

A. Kashaf et. al. introduced TSEP: Threshold-sensitive stable election protocol for WSNs. TSEP [13] is a imprudent, threshold based protocol that increase steadiness and lifespan of the network.

S. Kumar et al. extended the previous SEP based protocols and proposed an energy aware reactive routing protocol with 3 dissimilar types of nodes i.e. advanced nodes, transitional nodes and normal nodes. Protocol was named as Enhanced Threshold Sensitive Stable Election Protocol for Heterogeneous Wireless Sensor Network ETSSEP [14]. The election probabilities are governed by remaining node energy and aggregate network energy at round $\mathrm{r}$.

Threshold for a node to be elected as a $\mathrm{CH}$ is calculated as:-

$$
T(n)=\left\{\begin{array}{l}
\frac{p}{1-p \bmod \left(r, \frac{1}{p}\right)} * \frac{\text { Re sidual Energy of node }}{\text { Average Energy of Network } K_{\text {opt }}} \quad \text { if } n \in G \\
0 \quad \text { otherwise }
\end{array}\right.
$$

A similar 3 tier approach is seen in (ESEP) An Enhanced Stable Election Protocol proposed by Femi A. Aderohunmuet. al.[15] Authors claim that nodes with the highest energy will always be the preferred candidate for the cluster head, but the problem of abrupt size of the clusters is yet not addressed.

Younis et al. supplemented the LEACH protocol and designed hybrid energy efficient distributed (HEED) clustering protocol [16]. It defines the possibility of a node to be chosen as a $\mathrm{CH}$ :

$$
C H_{\text {prob }}=C_{\text {prob }} * \frac{E_{r}}{E_{\max }}
$$

Where Er is the present remaining node energy and Emax is the highest initial energy which was same for all the nodes. It was successful in achieving a drop in messages being exchanged. Nonetheless, this protocol does not assume any distribution of the nodes or location awareness.

Another family of clustering based protocols originates from DEEC [17], proposed by L. Qing et. al. DEEC comprehended the ideology of LEACH [3] and HEED [16]. It works on multiple heterogeneous networks setting where all the nodes have different energy levels. $\mathrm{CHs}$ are elected per round depending on the weighted likelihood of fraction of remaining energy and aggregate network energy. The rotating epoch is calculated as

$$
n_{i}=p_{o p t} * \frac{E_{r_{i}}}{E_{a}}
$$

Where Eri is remaining energy of each node and Ea is aggregate network energy.

To accommodate heterogeneous settings, standard probability pi of a node to be elected as $\mathrm{CH}$ in every ni rounds is given by:

$$
p_{i}=\frac{p^{*} n^{*}(1+a) E_{i}}{\left(n+\sum_{i=1}^{n} a_{i}\right) * E_{a}}
$$

The authors claim to have achieved a uniform dissemination of energy depletion amongst the nodes, but it is observed that there is a high variation in no. of $\mathrm{CH}$ nodes per round and node degree of CHs. The results depict that DEEC outperforms LEACH and SEP. Therefore for this paper DEEC has been taken as a benchmark for comparing results.

Versions of DEEC like DDEEC [18], E-DEEC (Enhanced Distributed Energy Efficient Clustering) [19], ipEDEEC [20], EDDEEC [21] and hetDEEC [22]: heterogeneous distributed energy efficient clustering protocol diversified the approach further and implements the network with three-level heterogeneity using weighted election probability. The authors claim to enhance the energy levels and the network lifetime. But none of them ensured the even distribution of nodes in the clusters. 
Arati Manjeshwar et al. introduced a Threshold sensitive Energy Efficient sensor Network protocol TEEN [23] for networks which permit intermittent variations in the significant factors of the network like threshold. The sensors continuously monitor the environment and transmit data only when hard threshold (HT ) and soft threshold (ST )criteria is met. Initially a node transmits data only if currently sensed value crosses the hard threshold limit. Eventually the node transmits data only if the change in currently sensed value and last sensed value exceeds soft threshold. After every round values of ST and HT can be adjusted to have control over the tradeoff between energy efficiency and accuracy. This approach however suffers a problem of lack of communication amongst nodes if thresholds are never reached. In that case base station might not receive any updates from the network.

Arati Manjeshwar and D. Agrawal proposed Adaptive Threshold-sensitive Energy Efficient Network (APTEEN) [24], as an enhancement over TEEN [23]. CHs were assigned the task of sharing necessary parameters like threshold, TDMA schedule and a time counter (for controlling collisions). However, in this approach nodes die prematurely because of overburdening of $\mathrm{CHs}$.

J. Ma and S. Wang distributed the load of CH's in Adaptive Threshold-sensitive Energy Efficient Network (ADCAPTEEN) [25] and Multiple Adaptive Thresh-old-sensitive Energy Efficient Network based on Ant-colony (AMAP-TEEN) [26]. The authors suggest using two $\mathrm{CHs}$, one master $\mathrm{CH}(\mathrm{MCH})$ and one vice $\mathrm{CH}(\mathrm{VCH})$ per cluster that will work simultaneously on data gathering, synthesis and communication. $\mathrm{MCH}$ collects the data from all the cluster members as per their TDMA schedule, processes the data and elects that member node on the link as VCH which has the highest pheromone absorption. VCH then communicates directly with Base Station. In comparison to APTEEN [24] its successors, ADCAPTEEN [25] and AMAP-TEEN [26] shows improved node survival rate, less energy loss and good scalability but still they suffer from a lot of overhead and complications in the $\mathrm{CH}$ election process.

The work seen so far in literature, focuses on optimizing energy levels and enhancing network lifetime. Addressing one issue compromises the other attributes like complexity, time taken, redundancy etc. Some of the shortcomings of the above protocols which are addressed in this paper are discussed in the next section.

Here a resilient technique is proposed for heterogeneous clustered network which guarantees scalable and optimal performance of the network till the end of the network lifetime.

\section{Radio Energy Dissipation Model}

Though various radio models exist in literature, here first order radio model (Fig. 1) is used.

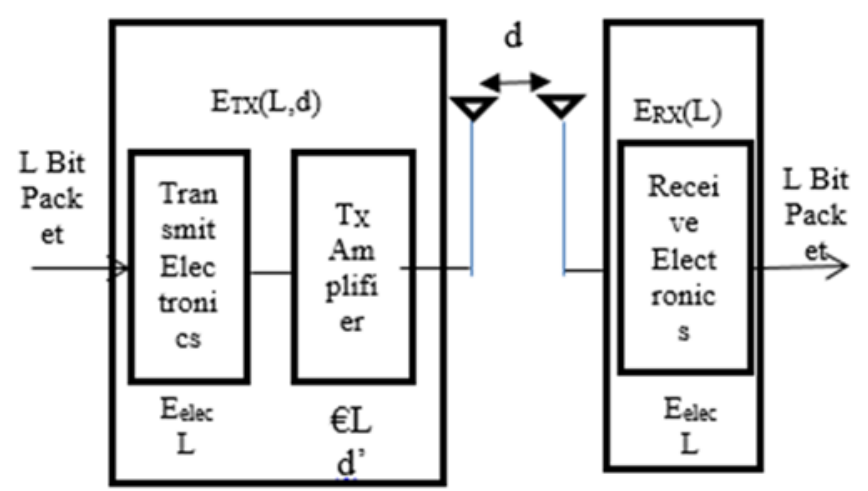

Figure1: Radio Energy Dissipation Model

In order to send a L-bit message over a distance d, Energy consumed by transmitter of the radio is given by:-

$$
E_{T X}(L, d)=\left\{\begin{array}{ll}
L \cdot E_{\text {elec }}+L \cdot E_{f s} \cdot d^{2} & \text { if } d<d_{0} \\
L \cdot E_{\text {elec }}+L \cdot E_{m p} \cdot d^{4} & \text { if } d \geq d_{0}
\end{array} \ldots \ldots \ldots\right.
$$

Where $\mathrm{E}_{\mathrm{elec}}$ is the energy consumed for each bit by the transmitter $\left(\mathrm{E}_{\mathrm{tx}}\right)$ and other receiver circuit $\left(\mathrm{E}_{\mathrm{rx}}\right)$. $\mathrm{E}_{\mathrm{elec}}$ relies on device specific features such as the digital coding, the modulation, the filtering, and the spreading of the signal [18]. For the experiments described here, transmitter amplifier model uses both the free space $\mathrm{E}_{\mathrm{fs}}$ with $\mathrm{d}^{2}$ power loss and the multi path fading $\mathrm{E}_{\mathrm{mp}}$ with $\mathrm{d}^{4}$ power loss channel models.

Threshold for distance between sender and receiver is calculated as:-

$$
d_{o}=\sqrt{\frac{E_{f s}}{E_{m p}}}
$$


If the distance (d) is less than $d_{0}$, the free space (fs) model ( $d^{2}$ power loss) is used otherwise; the multi path (mp) model ( $\mathrm{d}^{4}$ power loss) is used. Energy consumed by receiver can be expressed as

$$
E_{R X}= \begin{cases}L . E_{\text {elec }} & \text { for norma lnodes } \\ L .\left(E_{\text {elec }}+E_{D A}\right) & \text { for } C H^{\prime} s\end{cases}
$$

where $\mathrm{E}_{\mathrm{DA}}$ is data aggregation energy.

\section{Proposed Work}

This section is divided into two parts 4.1 Protocol Design and Analysis and 4.2 Simulation and results.

\subsection{Protocol Design}

As proposed in DEEC [17] and its variants, the network has multiple levels of heterogeneity where initial energy levels of nodes vary from Eo to $\mathrm{Eo}\left(1+\mathrm{a}_{\max }\right)$. Overall preliminary energy of the network can be equated as:

$$
E_{t}=\sum_{i=1}^{n} E_{o}\left(1+a_{i}\right)
$$

From eqn [4] it is clear that the nodes have definitely more energy levels and better processing capabilities. This heterogeneity of nodes increases the capacity of the whole sensor network. The Base Station (BS) is kept in the middle of the plot to maintain the uniformity of distance.

\subsubsection{Cluster Head (CH) Selection}

Clustering becomes inherent to any sensor network as the network size grows. Generally in this phase every node nominates itself for becoming a $\mathrm{CH}$ once every $1 / \mathrm{p}$ round. DEEC [17] uses variable epoch in every round. Probability threshold is calculated as given by [LEACH [3], HEED [16], SEP [12] , DEEC[17] etc.

$$
T(n)= \begin{cases}\frac{p}{1-p \bmod \left(r, \frac{1}{p}\right)} & \text { if } n \in G \\ 0 & \text { otherwise }\end{cases}
$$

But in the proposed protocol MEC (Maximum Energy Clustering), a greedy approach is used for clustering. After basic initialization of the network, the optimal probability of number of Cluster Heads (OCHs) is calculated on the basis of number of alive nodes.

$$
\text { OCHs }=p^{*} \text { alive }
$$

Where $\mathrm{p}$ denotes the possibility of $\mathrm{CHs}$, alive is no. of alive nodes and OCHs stands for the optimal probability of number of CHs. So if $\mathrm{p}=0.1$ and total alive nodes in the network at any point of time is 60 , optimal number of CHs will become 6

Nodes with maximum residual energies are selected as $\mathrm{CHs}$ for that round and information is relayed to the Base Station (BS). CHs are responsible for collecting, combining and relaying information to the BS. Energy dissipation is calculated as explained above in first order radio model.

\subsubsection{Association of Nodes with $\mathrm{CH}$}

In this phase all the normal nodes decide either they wish to communicate directly with BS or they would attach themselves to nearest $\mathrm{CH}$ (based on distance). All the nodes associated with the $\mathrm{CH}$ send their data to $\mathrm{CH}$. However a large amount of this data is highly repetitive.

As sensors record and send data at an extremely high rate, all the data send is not of much importance. In the proposed approach (MEC), the redundant data sent by the nodes to the respective $\mathrm{CHs}$ is not taken into account. The communication between a normal node $\mathrm{j}$ and respective $\mathrm{CH}$ is subjected to some predetermined thresholds.

Normal nodes sense the environment and record the value. (A random sensor value in a fixed range is generated here).

$$
G(j) . c s v=\operatorname{Rand}() ;
$$

Where $G$ is the network, $j$ is the node under consideration, csv stands for currently sensed value.

Two thresholds (Hard Threshold $\mathrm{T}_{\mathrm{h}}$, Soft Threshold $\mathrm{T}_{\mathrm{s}}$ ) are considered here as in [11], [23] for activating the intra cluster communication. If the currently sensed value (csv) crosses the Hard Threshold limit and the change between previously sensed value and currently sensed value crosses the Soft Threshold limit then the node finds and attaches itself with the nearest $\mathrm{CH}$. The intra cluster communication starts and the energy levels are updated accordingly. 


\subsubsection{Pseudo Code and Flowchart}

Pseudo code (Fig. 2) and flowchart (Fig. 3) for the algorithm used in the above process are given below.

$$
\text { Pseudocode }
$$

$\operatorname{MEP}(S, n, p)$

Input :S-Set of Nodes,

$n-$ No. of Nodes,

p-optimal probability of $\mathrm{CHs}$

1. Start

2. $G \leftarrow S$

3. Sort G.E

4. Calculate $\mathrm{OCHs} \leftarrow P^{*} n$

5. for all i in $\mathrm{OCHs}$

6. $C H[i] \leftarrow G[i]$

7. Update $G[i] . E$

8. $P_{B S} \leftarrow P_{B S}+1$

9. end

10. For all $j \in(n-O C H s-$ dead $)$

$11 . G(j) . c s v \leftarrow G[j]$. Sense $\leftarrow \operatorname{Rand}()$;

12. if $\left(G(j) . c s v>=T_{h}\right)$

13. if $\left(a b s(G(j) . c s v-G(j) . p s v)>=T_{S}\right)$

14. Find $c \leftarrow$ nearest cluster () ;

15. $P_{C H} \leftarrow P_{C H}+1$

16.Update $G(j) . E, G(c) . E$

17. If $(G(j) . E<=0)$

18. $G(j) . E \leftarrow$ Dead

19. $(G(c) \cdot E<=0)$

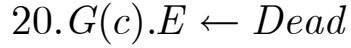

21. $G(j) . p s v \leftarrow G(j) . c s v$

22. end

23. end

24. end

25. stop

Fig. 2 : Pseudo Code of MEC 


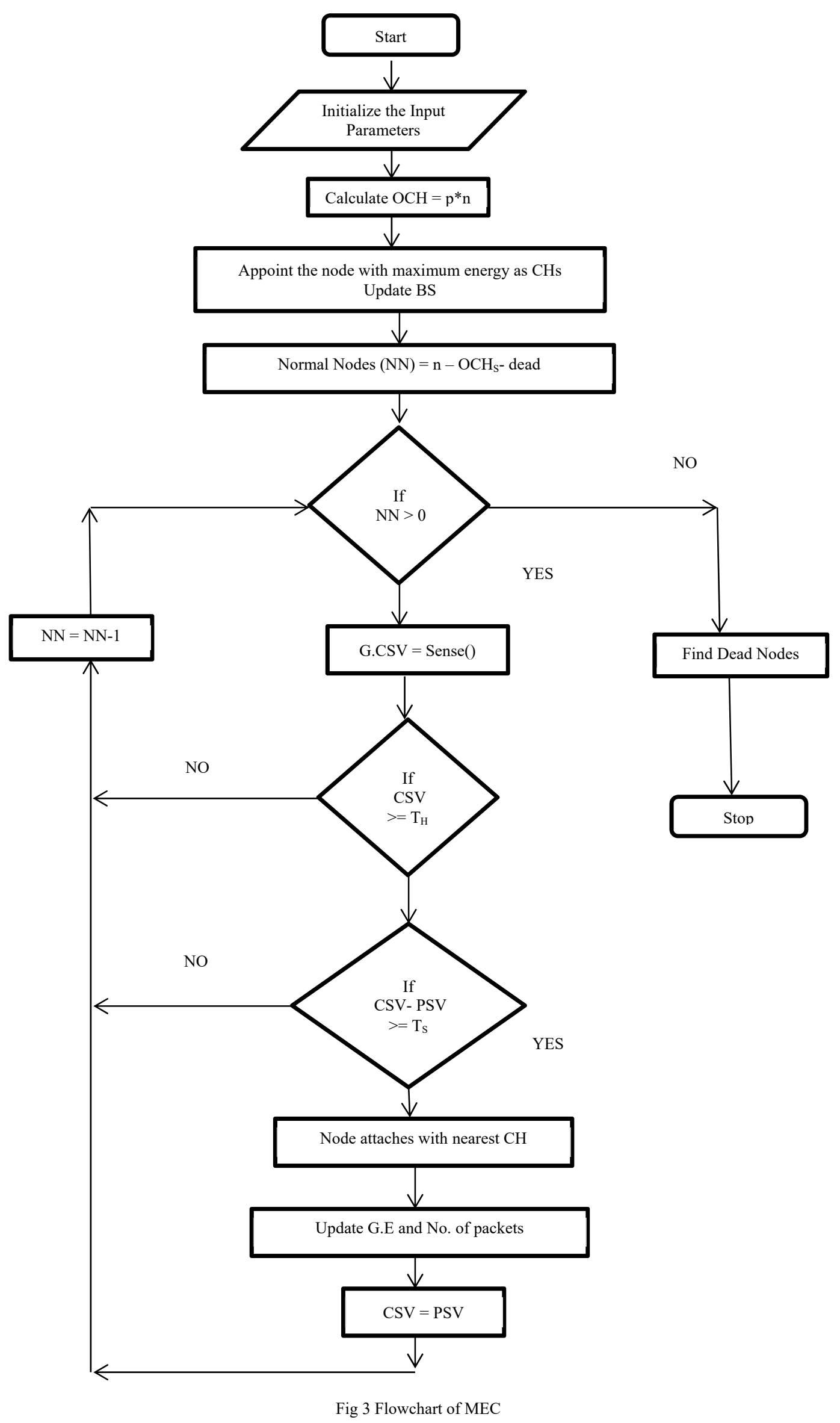


The proposed protocol, MEC starts with the nodes with highest energies acting as Cluster Heads (CHs). CHs then informs the Base Station (BS). Whereas in its counterpart DEEC, a random node is elected to be a $\mathrm{CH}$ and total number of $\mathrm{CHs}$ in a round is also arbitrary. Here the ideal number of CHs in a network in a particular round is determined by the count of alive nodes in the network and a predefined percentage which can be varied. The remaining nodes act as normal nodes $(\mathrm{NN})$. Normal nodes find a $\mathrm{CH}$ which is nearest to them (on the basis of Euclidean distance between a node, BS and a $\mathrm{CH}$ ) and transmit their recordings to the respective $\mathrm{CH}$ if the observation is above a certain threshold. In case a node is nearer to BS it can directly communicate with BS.

Thereafter the energies of sender and receiver are updated. In the next round all the nodes are sorted (descending order) on the grounds of their updated residual energies. Again a percentage of nodes with highest remaining energies are designated to be $\mathrm{CHs}$, normal nodes attaches themselves with either one of the $\mathrm{CH}$ or $\mathrm{BS}$ itself. The process continues until there are alive nodes in the network. Results section shows that MEC has higher stability period and longer network lifetime.

\subsection{Simulation and Results}

This section shows the simulation results of newly proposed protocol MEC (Maximum Energy Clustering) and its comparison with DEEC on the same environmental and network setting.

In the experiment, 150 nodes are arbitrarily positioned in an area of $100 \times 100$. A Base station (BS) or sink is fixed in the center of the plot. Sensor nodes are spatially dispersed and deterministically deployed on the field.

Simulation parameters and other radio characteristics are given in Table 1.

Table: 1 Simulation Parameters

\begin{tabular}{|l|l|}
\hline Parameter & Value \\
\hline $\mathrm{N}$ & 150 \\
\hline Sink.x, Sink.y & 50,50 \\
\hline $\mathrm{R}_{\max }$ & 3000 \\
\hline $\mathrm{P}$ & 0.1 \\
\hline $\mathrm{x}_{\mathrm{m}} \mathrm{x}_{\mathrm{m}}$ & $100 \mathrm{x} 100$ \\
\hline $\mathrm{E}_{\text {elec }}$ & $5 \mathrm{~nJ} / \mathrm{bit}$ \\
\hline $\mathrm{E}_{\mathrm{fs}}$ & $10 \mathrm{pJ} / \mathrm{bit} / \mathrm{m}^{2}$ \\
\hline $\mathrm{E}_{\mathrm{mp}}$ & $0.0013 \mathrm{pJ} / \mathrm{bit}^{2} / \mathrm{m}^{4}$ \\
\hline $\mathrm{E}_{0}$ & $0.5 \mathrm{~J}$ \\
\hline $\mathrm{E}_{\mathrm{DA}}$ & $5 \mathrm{~nJ} / \mathrm{bit} / \mathrm{message}$ \\
\hline Sensor Range & $50-200$ \\
\hline $\mathrm{T}_{\mathrm{h}}$ & 100 \\
\hline $\mathrm{T}_{\mathrm{s}}$ & 2 \\
\hline $\mathrm{L}$ & $4000 \mathrm{bits}$ \\
\hline
\end{tabular}

Matlab Platform is used for the performance evaluation of both the protocols MEC and DEEC in terms of dead nodes, alive nodes, number of data packets sent to $\mathrm{CHs}$, No. of packets sent to BS and the No. of cluster Heads in every round.

General observations are summarized in Table 2.

Table 2: Comparison between MEC and DEEC

\begin{tabular}{|l|l|l|}
\hline Parameter & MEC & DEEC \\
\hline First Dead & 2674 & 1251 \\
\hline Tenth dead & 2684 & 1450 \\
\hline All Dead & 2696 & 2925 \\
\hline Packets to BS & 56816 & 71719 \\
\hline Packets to CH & 225633 & 216091 \\
\hline Total CHs & 40332 & 22795 \\
\hline
\end{tabular}


From the above Table- 2 it can be observed that, at the end of simulation, in MEC, there is a double fold increase in the time elapsed by the first and the tenth node to die. However, "All the nodes" in MEC die earlier than DEEC but it is because of stochastic distribution of CHs. The performance of MEC is consistent till the end of the network until all the nodes exhaust to their fullest capacity.

In the earlier used techniques mentioned in literature [3], [12], [16], [17], the sizes of clusters varies briskly. This leads to congestion and packets drop during intra cluster communication. However in $\mathrm{MEC}, \mathrm{CH}$ selection relies on the nodes alive in the network at given point of time and the remaining energies of the nodes. This evenly distributes the number of $\mathrm{CHs}$ in each round [Fig. 4].

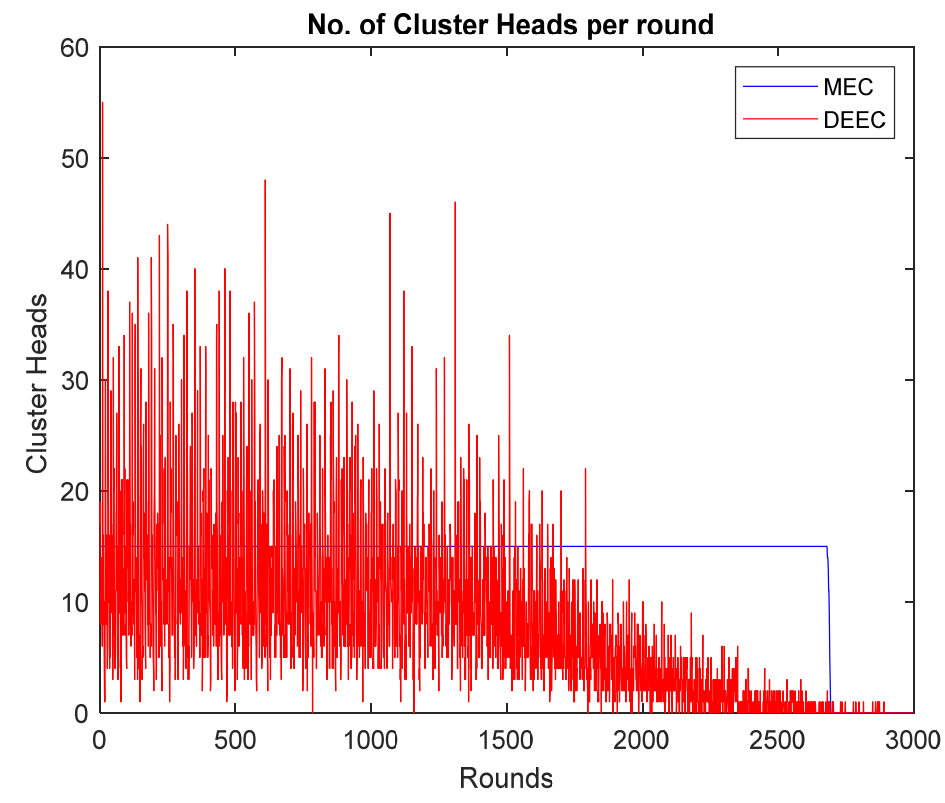

Fig: 4- No. of Cluster Heads per round

Though the distance between the nodes is not yet considered while selecting $\mathrm{CH}$ (will be considered in future work), sizes of the clusters are still manageable and does not vary abruptly. The node degree (No. of normal nodes associated with a $\mathrm{CH}$ ) of $\mathrm{CHs}$ in MEC vary from 14 to 1 whereas it was 50 to 1 in its counterparts [3], [17], [23]. This improvises the throughput and reduces end to end delay in the whole network.

As shown in [Fig. 4], in MEC, the $\mathrm{CH}$ count decreases only towards the end of the simulation. It decreases single hop communication of a normal node with BS. A significant drop is seen in No. of packets going to BS in MEC as compared to DEEC. [Fig. 5]

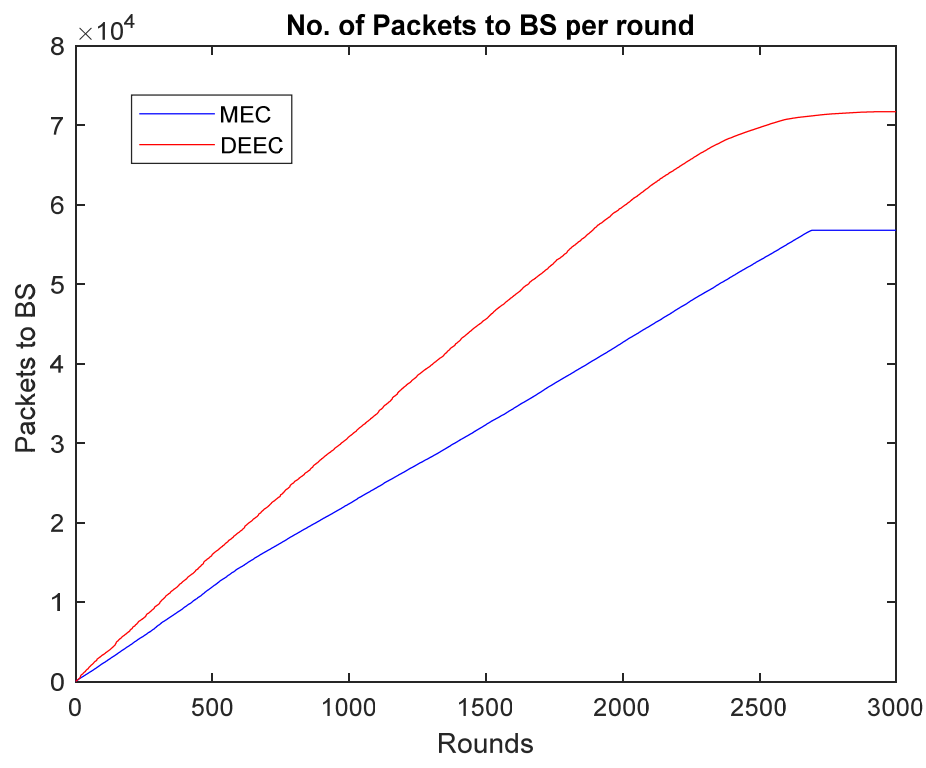

Fig: 5- No. of packets to BS per round 
This results in energy preservation of normal nodes. Total energy of the whole network increases giving rise to more number of alive nodes (Fig. 7) and less number of dead nodes (Fig. 6) per round. The transition from alive to dead nodes in MEC varies from 100 to 200 rounds. Unlike DEEC where the first node dies at approximately 1100 th round and the last node dies at 3000 th round. In the suggested protocol, MEC, the stability period (i.e. the time between the starting of execution and the time when the first node dies) increases. All the nodes are utilized uniformly on the basis of their remaining energies as only the nodes with highest energies get a chance to become $\mathrm{CH}$.

This in turn enhances network lifetime and increases sustainability of the network.

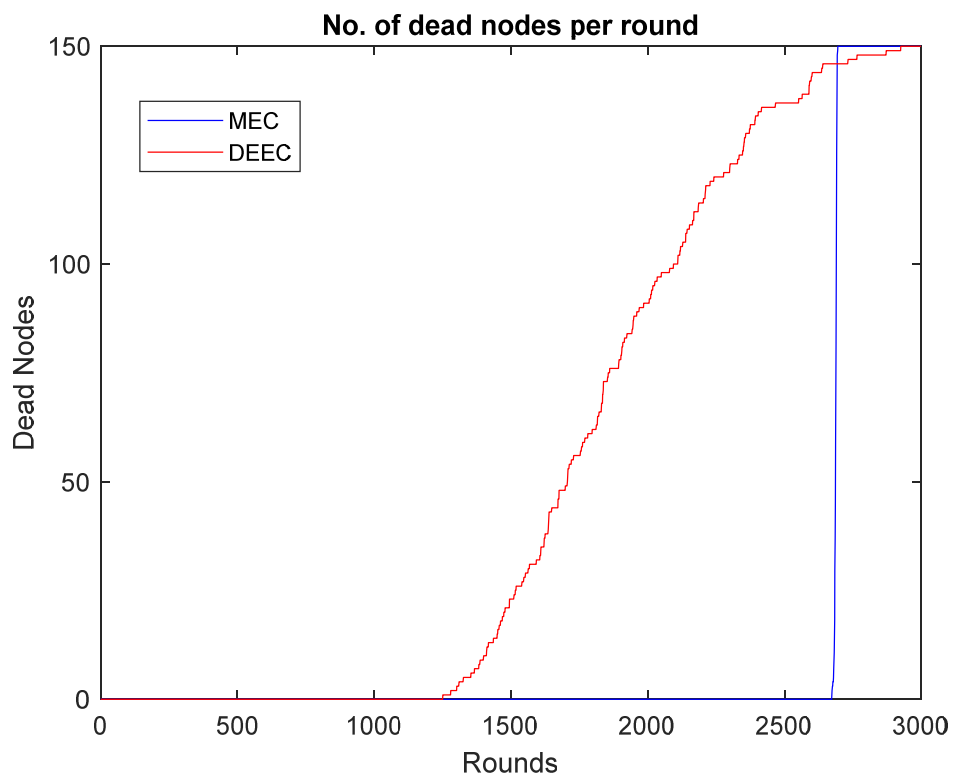

Fig: 6 - No. of dead nodes per round

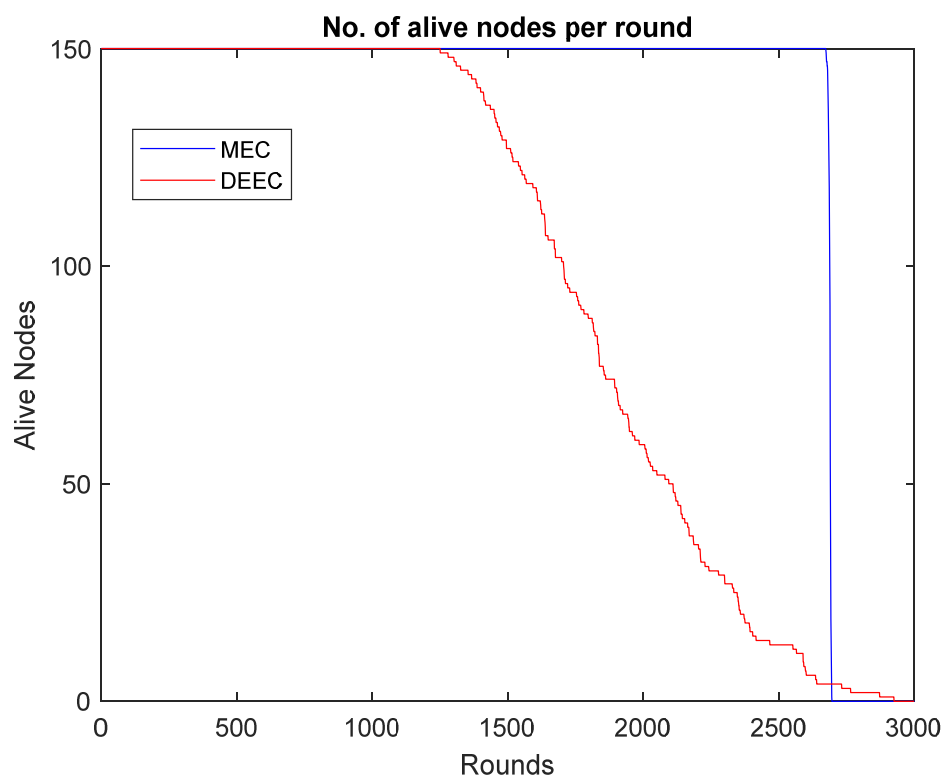

Fig: 7 - No. of alive nodes per round

In the proposed MEC protocol No. of packets being transmitted to $\mathrm{CHs}$ are controlled. Redundant data is identified by the comparison of sensing thresholds and is not transmitted. Results of MEC are comparable with that in DEEC as shown in (Fig. 8). 


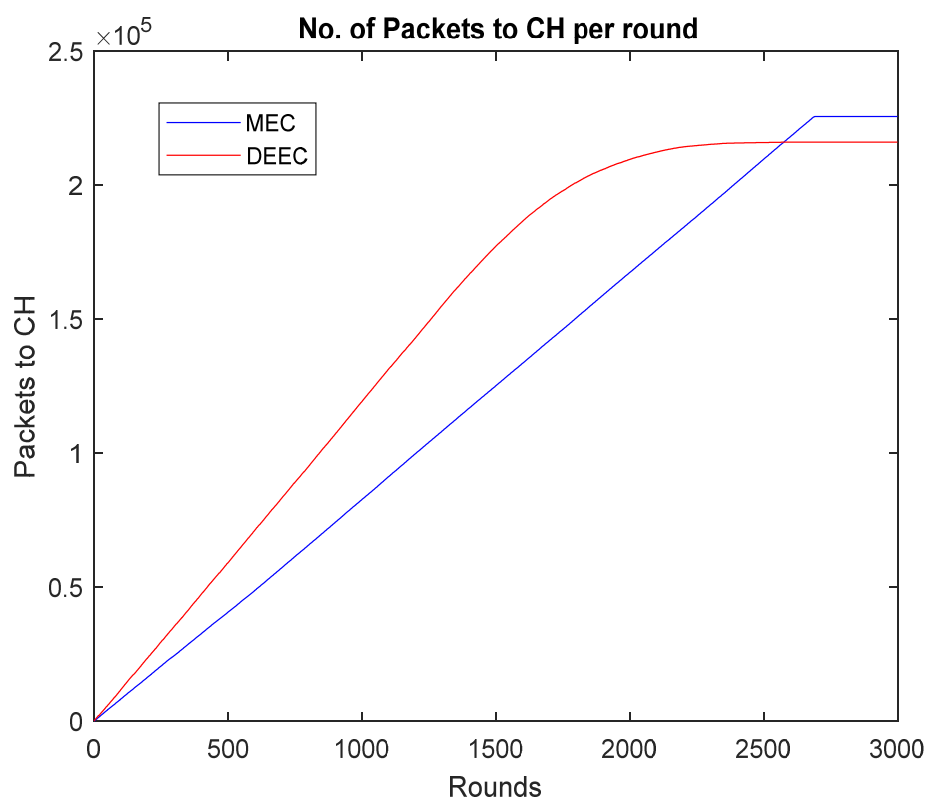

Fig: 8 No. of packets to $\mathrm{CH}$ per round

Total no. of clusters in MEC increases drastically but it can be attributed to robustness and stability of the network in accordance with performance, throughput, packet delivery ratio and accuracy.

\section{Conclusion and Future Directions}

Dense Wireless Sensor Networks deployed in otherwise difficult to reach areas, employ clustering as the vital process to administer the underlying complex structure. Sometimes the network is skewed and sometimes it is well distributed. To cater to all types of networks, clustering protocols should be robust and extensible. It should be able to provide integral and timely information. The proposed protocol MEC is able to attain a significant reduction in network traffic besides utilizing the network to its fullest capacity. Results are compared to a wellknown protocol DEEC and it is examined that MEC outperforms DEEC as it follows a fair $\mathrm{CH}$ election process. Though the distance between the nodes is not yet considered while selecting $\mathrm{CH}$, sizes of the clusters are still manageable and do not vary abruptly. The future work will focus on stabilizing the network load of CHs. MEC decreases single hop communication of a normal node with BS. A

Significant drop is seen in No. of packets going to BS in MEC as compared to its counterpart DEEC

\section{References}

[1] A. Garg and M. Jailia, "Addressing Data Redundancy in IoT Networks", International Journal of Recent Technology and Engineering, vol. 8, no. 4, pp. 8000-8004, 2019.

[2] A. Garg and M. Jailia, "Mining Iot Data for Next Generation Smart Cities", International Journal of Engineering and Advanced Technology, vol. 8, no. 6, pp. 259-262, 2019.

[3] W. Heinzelman, A. Chandrakasan, and H. Balakrishnan, "Energy-efficient routing protocols for wireless microsensor networks," in Proc. 33rd Hawaii Int. Conf. System Sciences (HICSS), Maui, HI, Jan. 2000.

[4] Fan Xiangning1, Song Yulin "Improvement on LEACH Protocol of Wireless Sensor Network" International Conference on Sensor Technologies and Applications 2007.

[5] M. Tong and M. Tang, "LEACH-B: An improved LEACH protocol for wireless sensor network," in Proc. 6th Int. Conf. Wireless Commun. Netw. Mobile Comput. (WiCOM), Sep. 2010, pp. 1-4.

[6] T. Voigt, A. Dunkels, J. Alonso, H. Ritter, and J. Schiller, "Solaraware clustering in wireless sensor networks," in Ninth International Symposium on Computers and Communications. vol. 1, June 2004, pp. 238-243.

[7] C. Divya,, N. Krishnan, and A Petchiammal, "Modified low energy adaptive clustering hierarchy for heterogeneous wireless sensor network," in International Journal of Engineering \& Technology, Vol. 5, No. 2, pp.536-541, 2013.

[8] Abdelhamied A. Ateya, Mohammed S. Sayed and Mahmoud I. Abdalla, "Multilevel Hierarchical Clustering protocol for wireless sensor networks", International Conference on Engineering and Technology (ICET), 19-20 April 2014

[9] M. O. Farooq, A. B. Dogar, and G. A. Shah, "MR-Leach: Multi-hop routing with low energy adaptive clustering hierarchy," in Proc. 4th Int. Conf. Sensor Technol. Appl. (SENSORCOMM), Jul. 2010, pp. 262-268.

[10] B. Manzoor, N. Javaid, O. Rehman, M. Akbar, Q. Nadeem, A. Iqbal, M. Ishfaq, "Q-LEACH: A New Routing Protocol for WSNs", International Workshop on Body Area Sensor Networks (BASNet-2013) in Conjunction with 4th International Conference on Ambient Systems,Networks and Technologies (ANT 2013), 2013, Halifax, Nova Scotia,Canada, Procedia Computer Science, Volume 19, 2013, Pages 926-931, ISSN 1877-0509.

[11] D. Mahmood, N. Javaid, S. Mahmood, S. Qureshi, A. M. Memon, and T. Zaman, “MODLEACH: A variant of LEACH for WSNs," in Proc. 8th Int. Conf. Broadband Wireless Comput., Commun. Appl. (BWCCA), Oct. 2013, pp. 158-163.

[12] I. Matta, G. Smaragdakis, and A. Bestavros, "SEP: A stable election protocol for clustered heterogeneous wireless sensor networks," in SANPA, August 2004.

[13] Aasia Kashaf, Nadeem Javaid, Zahoor Ali Khan and Imran Ali Khan, “TSEP: Threshold-sensitive Stable Election Protocol for WSNs”, 
$10^{\text {th }}$ IEEE International Conference on Frontiers of Information Technology (FIT' 12), 2012, Pakistan.

[14] Kumar, S., Verma, S.K., Kumar, A.: 'Enhanced threshold sensitive stable election protocol for heterogeneous wireless sensor network', Wirel. Pers. Commun., 2015, 85, (4), pp. 2643-2656.

[15] Femi A. Aderohunmu, Jeremiah D. Deng, “An Enhanced Stabel Election Protocol (SEP) for Clustered Heterogeneous WSN", Discussion Paper Series, No. 2009/07, October 2009, ISSN: 1177-455X, Department of Information Science, University of Ontago, (2010).

[16] O. Younis and S. Fahmy, "HEED: A Hybrid, Energy-Efficient, Distributed Clustering Approach for Ad Hoc Sensor Networks", IEEE Transactions on Mobile Computing, vol. 3, NO. 4, pp. 366-379, Oct-Dec 2004.

[17] L. Qing, Q. Zhu, M. Wang, "Design of a distributed energy-efficient clustering (DEEC) algorithm for heterogeneous wireless sensor networks". ELSEVIER, Computer Communications 29, 2006, pp 2230- 2237.

[18] B. Elbhiri, R. Saadane, S. El fldhi, and D. Aboutajdine, "Developed distributed energy-efficient clustering (DDEEC) for heterogeneous wireless sensor networks," in Proc. 5th Int. Symp. I/V Commun. Mobile Netw. (ISVC), Sep. 2010, pp. 1-4

[19] P. Saini and A. K. Sharma, "E-DEEC- enhanced distributed energy efficient clustering scheme for heterogeneous WSN," in Proc. 1st Int. Conf. Parallel Distrib. Grid Comput. (PDGC), Oct. 2010, pp. 205-210.

[20] A. Saini, A. Kumar, H. Mandoria and B. Pandey , (2016). "Study and analysis of DEEC protocols in hetergeneous WSNs using MATLAB" In International Research Journal of Engineering and Technology, e-ISSN- 2395 -0056, p-ISSN2395-0072, Vol. 3 Issue-8, Aug 2016

[21] N. Javaid, T. Qureshi, A. Khan, A. Iqbal, E. Akhtar, and M. Ishfaq, "EDDEEC: Enhanced developed distributed energy-efficient clustering for heterogeneous wireless sensor networks," 4th International Conference on Ambient Systems, Networks and Technologies (ANT 2013), 2013, Halifax, Nova Scotia, Canada, Procedia Computer Science, Volume 19, 2013, Pages 914-919.

[22] S. Singh and A. Malik "hetDEEC: Heterogeneous DEEC Protocol for Prolonging Lifetime in Wireless Sensor Networks", Journal of Information and Optimization Sciences, vol. 38, no. 5, pp. 699-720, 2017.

[23] A. Manjeshwar and D. P. Agarwal, "TEEN: a Routing Protocol for Enhanced Efficiency in Wireless Sensor Networks," 1st Int'l. Wksp. on Parallel and Distrib. Comp. Issues in Wireless Networks and Mobile Comp., April 2000.

[24] A. Manjeshwar, D. Agrawal, "APTEEN: A Hybrid Protocol for Efficient Routing and Comprehensive Information Retrieval in Wireless Sensor Networks," In Proc. International Parallel and Distributed Processing Symposium, Florida, 2002, pp. 195-202.

[25] J. Ma, S. Wang, C. Meng, Y. Ge, and J. Du, "Hybrid energy-efficient APTEEN protocol based on ant colony algorithm in wireless sensor network,” EURASIP J. Wireless Commun. Netw., vol. 102, pp. 1-13, Dec. 2018.

[26] J. Ma, S. Wang, and Y. Ge, "Ant-Colony Based Double Cluster Heads Adaptive Periodic Threshold-Sensitive Energy Efficient Network Protocol in WSN," Lecture Notes in Electrical Engineering Communications, Signal Processing, and Systems, pp. 309-317, 2017.

[27] H. Zhang, S. Zhang, and W. Bu, "A Clustering Routing Protocol for Energy Balance of Wireless Sensor Network based on Simulated Annealing and Genetic Algorithm," in InternationalJournal of Hybrid Information Technology Vol.7, No.2, PP. 71-82, 2014.

[28] Guravaiah, Koppala, Arumugam Kavitha, and Rengaraj Leela Velusamy. "Data Collection Protocols in Wireless Sensor Networks." Wireless Sensor Networks-Design, Deployment and Applications. IntechOpen, 2020.

[29] A. Dwivedi and A. Sharma, "FEECA: Fuzzy based Energy Efficient Clustering Approach in Wireless Sensor Network," EAI Endorsed Transactions on Scalable Information Systems, 2020. 